\title{
Correlation of Uterine Myoma to Menorrhagia
}

\author{
Poonam Sharma, Ashma Rana, Kesang Diki Bista. \\ Department of Obstetrics \& Gynaecology, TU Teaching Hospital, Kathmandu.
}

\begin{abstract}
Aim: The major objective was to find out the correlation between site and size of uterine myoma to menorrhagia. Methods: This was a prospective descriptive study which was conducted at department of Obstetrics and Gynaecology, Tribhuvan University Teaching Hospital, Kathmandu. Intra-operative findings and symptomatology of 70 consecutive women who underwent surgery for uterine myoma from March 2005 to October 2005 were evaluated.

Results: The mean age of the women was $45.0 \pm 5.3$ years. Intramural myoma was the most common type, occurring in $44(62.85 \%)$ women at single site and in $20(28.57 \%)$ in combinations with other types of myoma whereas submucous and broad ligament myoma were the least common occurring in 2 (2.9\%) and $1(1.4 \%)$ women respectively at single site. Menorrhagia was observed in $58.6 \%$ of women. The mean size of myoma was $63.2 \pm 79.9 \mathrm{~cm}^{2}$. There was increased relative risk of menorrhagia $(\mathrm{RR}=1.74,95 \% \mathrm{Cl} 1.42$ $2.14)$ in submucosal types and the risk did not increase with an increase in area of $>50 \mathrm{~cm}^{2}$ of myoma; however menorrhagia did not correlate significantly with site and size of myoma ( $p$-value $>0.05)$. It was concluded that there was no significant correlation between site and size of myoma and menorrhagia ( $p$ value $>0.05$ ), although the risk of menorrhagia was increased in submucosal type.
\end{abstract}

Key words: Myoma /Leiomyoma, Menorrhagia, Hysterectomy, Fibroid uterus,

\section{Introduction}

Uterine myoma commonly known as fibroid is the commonest benign pelvic tumours involving myometrial smooth muscle and extra cellular matrix (proteins, collagen and elastin) in female and forms the commonest indication for hysterectomy. Uterine myoma affects millions of women and accounted for 60 percent of the 600,000 hysterectomies annually performed in the United States ${ }^{1}$. Menorrhagia is the most frequent complaint related to myoma. Myomas, located at different sites, such as subserous, intramural, submucous, broad ligament, cervical either single or multiple are clinically apparent in up to $20 \%$ of women causing significant morbidity such as prolonged and heavy menstrual bleeding (menorrhagia) ${ }^{2}$. Menorrhagia is defined as the menstruation at regular cyclic intervals but with excessive flow and duration. Clinically it is defined as total menstrual blood loss exceeding $80 \mathrm{ml}$ per cycle or menstrual flow lasting longer than 7 days ${ }^{3}$. Menorrhagia has been quoted as the commonest symptom of myoma. However, there are conflicting reports regarding association of morphology of uterine myoma and menorrhagia ${ }^{4-6}$. Uterine artery embolization in myomas are up coming management that aims at conservation of the uterus, as these tumours are benign and affects women who are infertile. ${ }^{7-9}$.

In our Institute myoma forms the most common indication of hysterectomy and most of the surgeries for myoma are performed on large sized uterus with myoma of considerable sizes, often posing problems at its removal. Based on this finding we wanted to study why Nepalese women with myoma present so late. Whether it is due to the lower incidence of submucous myoma, which often produces symptoms earlier because of menorrhagia or it is due to poor healthseeking behaviour of Nepalese women because of unawareness.

We have also decided to conduct this study, as there have been no such studies in Nepal correlating the myoma with clinical symptoms. We believed that

Correspondence

Dr. Poonam Sharma FCPS

Teaching Assistanc, Department of Obstetrics \& Gynaecology, TU Teaching Hospital, Kathmandu.

punishita@yahoo.com,poonamrkoirala@yahoo.com 
menorrhagia may not be the commonest symptom because submucous myoma which produces menorrhagia often report earlier, where as in our clinical practice we often see large sized uterus at the time of presentation. This study aimed to rework on myth and truth of myoma, their common presentation to verify whether menorrhagia correlates with the size and site of myoma in view of controversial reports showing varied relationship between myoma morphology and menorrhagia.

\section{Methods}

This study was carried out at the Department of Obstetrics and Gynaecology of Tribhuvan University Teaching Hospital, Kathmandu, Nepal, from March 2005 till October 2005. All the cases undergoing hysterectomy/myomectomy for myoma were enrolled except those woman in whom myoma was diagnosed preoperatively and later found not to have myoma during surgery were excluded from the study including women who were found to have myoma during Caesarean section or woman with myoma undergoing hormonal treatment like OCP (Oral contraceptive pills), DMPA (Depot Medroxy-progesterone acetate), hormonal replacement therapy (HRT) and hormone containing IUCD (Intra uterine contraceptive device).

Type of uterine myoma, presence of menorrhagia and its duration, other common symptoms of uterine myoma and their durations, number of myoma in each patient and total area of all myomas in each patient were noted. Data were double entered into a personal computer and were analyzed using The Statistical Package for Social Sciences (SPSS) version 11.5 software (SPSS Inc, Chicago, IL). Data collected were reported as mean \pm standard deviation. Association between variables were tested using Chi-square test, Fisher's Exact test and student's $t$-test appropriately. A $p$ value of less than 0.05 was considered statistically significant.

\section{Results}

Of the 74 women admitted with a diagnosis of uterine myoma for elective surgery, 70 who had gross appearances of myoma operatively were enrolled for the study who had histo-pathologically proven leiomyoma uteri excluding four cases which instead looked like adenomyosis intraoperatively. Mean age of the women was $45.0 \pm 5.3$ years. Ninety seven percent of the women were married. Among these women, $85.7 \%$ were housewives and only $2(2.9 \%)$ were working. Similarly, $82.9 \%$ of women were from middle socio-economic class. The education level of these women was very low, as only $5(7.1 \%)$ women had done their graduation. In this study, women came for treatment of uterine myoma about 17 years after completion of their families. Mean age at marriage was
$20 \pm 2.0$ years and they were married for $26 \pm 5$ years in average before being admitted for surgery. Only 3 $(4.4 \%)$ out of 68 women were found to be infertile. The average number of pregnancies was $3.1 \pm 1.65$.

From the menstrual history of these women, it was found that 69 women were menstruating and one woman was postmenopausal. The mean duration of menstrual flow was $7 \pm 2.5$ days. According to definition of menorrhagia, 41 (59.4\%) out of 69 menstruating women were suffering from menorrhagia. In those 41 women, $38(55.1 \%)$ had menstrual flow that was prolonged (e 7 days) and another $3(4.3 \%)$ had normal duration of menstrual flow, but had heavy and excessive bleeding. They described their bleeding as gushing type and some reported bleeding with passage of excessive clots (Figure 1).

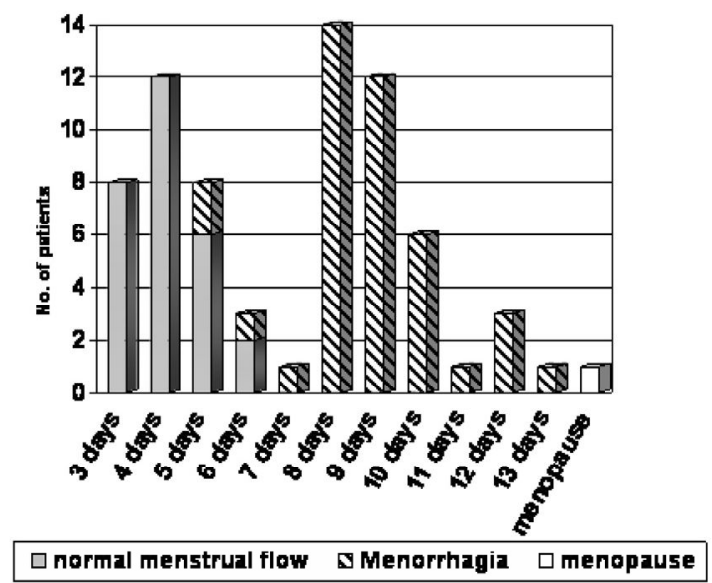

Fig 1. Duration of menstrual flow

Among 70 women, 69 underwent abdominal hysterectomy, whereas one woman underwent vaginal hysterectomy for uterine myoma. Different parameters were measured per-operatively, namely: uterine size in $\mathrm{cm}^{2}$, length of uterine cavity, total number of myoma, and total area of all the myoma in every case. The mean size of uterus before surgery was about $11.9 \pm 3.9$ weeks. The mean size of uterus after the surgery as per cutsection findings was $12.2 \pm 4.0 \mathrm{~cm}^{2}$.

Specimens were examined for types and number of myoma. The mean number of myoma was $2.67 \pm 2.16$. Only 27 (38.6\%) women had solitary myoma, whereas $43(61.4 \%)$ women had more than one myoma. Intramural myoma was found to be the most common type as it was present in $64(91.43 \%)$ out of 70 cases. Those intramural myoma were solitary in $21(30.0 \%)$ women and multiple in $23(32.86 \%)$ women. They occurred in combination in $20(28.57 \%)$ women with submucous, subserous or including both or with broad ligament types. Similarly the next common type of myoma was subserous which was present in $17(24.28 \%)$ cases. 
Table 1. Types of myoma and their occurrence

\begin{tabular}{|c|c|c|c|}
\hline \multicolumn{2}{|c|}{ Type of myoma } & \multirow{2}{*}{$\frac{\text { No. of patient }}{2}$} & \multirow{2}{*}{$\begin{array}{r}\text { Percentage } \\
2.9\end{array}$} \\
\hline Submucous $(n=2)$ & Solitary & & \\
\hline & Multiple & 0 & 0 \\
\hline \multirow[t]{2}{*}{ Intramural $(n=44)$} & Solitary & 21 & 30.0 \\
\hline & Multiple & 23 & 32.9 \\
\hline \multirow[t]{2}{*}{ Subserous $(\mathrm{n}=3)$} & Solitary & 3 & 4.3 \\
\hline & Multiple & 0 & 0 \\
\hline \multicolumn{2}{|c|}{ Submucous + Intramural } & 5 & 7.1 \\
\hline \multicolumn{2}{|c|}{ Submucous + Intramural + Subserous } & 1 & 1.4 \\
\hline \multicolumn{2}{|c|}{ Intramural + Subserous } & 13 & 18.6 \\
\hline \multicolumn{2}{|l|}{ Broad ligament } & 1 & 1.4 \\
\hline \multicolumn{2}{|c|}{ Broad ligament + Intramural } & 1 & 1.4 \\
\hline \multicolumn{2}{|l|}{ Total } & 70 & 100 \\
\hline
\end{tabular}

Table 2. Different types of myoma: same site or in combination and their relationship with occurrence of menorrhagia $(n=70)$

\begin{tabular}{lccc}
\hline Types of myoma & Total $(\mathbf{n}=\mathbf{7 0})$ & Menorrhagia(n=41) & p-value \\
\hline Submucous & $2(2.9 \%)$ & $2(100.0 \%)$ & 0.13 \\
Intramural & $44(62.9 \%)$ & $22(50.0 \%)$ & 0.22 \\
Subserous & $3(4.3 \%)$ & $0(0.0 \%)$ & 0.25 \\
Intramural + Submucous & $5(7.1 \%)$ & $4(80.0 \%)$ & 0.40 \\
Subserous + Intramural & $13(18.6 \%)$ & $11(84.6 \%)$ & 0.72 \\
Subserous + Intramural + Submucous & $1(1.4 \%)$ & $1(100.0 \%)$ & 1.0 \\
Intramural + Broad ligament & $1(1.4 \%)$ & $1(100.0 \%)$ & 1.0 \\
\hline Broad ligament & $1(1.4 \%)$ & $0(0.0 \%)$ & 0.41 \\
\hline
\end{tabular}

Table 3. Relation between single location of myoma and menorrhagia

\begin{tabular}{lccccc}
\hline Type of myoma & Occurrence $(\mathbf{n}=\mathbf{7 0})$ & Menorrhagia & p-value & RR & 95\% CI \\
\hline Submucous Myoma & 2 & 2 & 0.508 & 1.74 & $1.42-2.14$ \\
Intramural myoma & 44 & 22 & 0.198 & 0.77 & $0.53-1.13$ \\
Subserous myoma & 3 & 0 & - & - & - \\
\hline
\end{tabular}

RR: Relative risk, 95\% CI: 95\% confidence intervals.

Table 4. Relationship between size of myoma and menorrhagia

\begin{tabular}{lcccc}
\hline $\begin{array}{l}\text { Total area of all myoma } \\
\text { in a patient }\end{array}$ & Yes & Menorrhagia & Total & p-value \\
\hline $1-50 \mathrm{~cm}^{2}$ & $24(58.5 \%)$ & $12(41.4 \%)$ & $36(51.4 \%)$ & \\
$51-100 \mathrm{~cm}^{2}$ & $11(26.8 \%)$ & $15(51.7 \%)$ & $26(37.1 \%)$ & \\
$101-150 \mathrm{~cm}^{2}$ & $2(4.9 \%)$ & $1(3.4 \%)$ & $3(4.3 \%)$ & \\
$151-200 \mathrm{~cm}^{2}$ & $1(2.4 \%)$ & $1(3.4 \%)$ & $2(2.9 \%)$ & \\
$>200 \mathrm{~cm}^{2}$ & $3(7.3 \%)$ & $0(0 \%)$ & $3(4.3 \%)$ & \\
\hline Total & $41(100 \%)$ & $29(100 \%)$ & $70(100 \%)$ & 0.192 \\
\hline
\end{tabular}


They existed as solitary single myoma in $3(4.28 \%)$ cases and in remaining $14(20 \%)$ they occurred in combination with submucus and intramural myoma. Submucous myoma was present in total $8(11.42 \%)$ cases out of 70 women. Broad ligament myoma was the least common type of myoma (in 2 women). The occurrence of different types of myoma was shown in detail in Table 1.

Out of 70 women, 65 (92.8\%) women were symptomatic; where as remaining $5(7.1 \%)$ women were asymptomatic. The most common presenting symptom was per vaginal bleeding which occurred in $60(85.71 \%)$ women. This largely was menorrhagia in $41(58.57 \%)$ cases. Surprisingly, $10 \%$ of these women had menorrhagia alone as the presenting symptom. Menorrhagia was expression of all the submucosal myoma except one and also of intramural myoma (39 out of 64) occurring either singly or in combination with other subtypes.

Dysmenorrhoea was the next most common symptom and was present in 29(41.4\%) women, followed by continuous vaginal bleeding in $13(18.6 \%)$ women and pelvic heaviness in 13 (18.6\%) women. In this study, women were suffering from menorrhagia for quite a long period and it was about $34.7 \pm 37.9$ months. On the other hand, women who had continuous vaginal bleeding came for medical help early (mean duration $=2.3 \pm 2.2$ months).

It is interesting to note that 22 out of $44(50 \%)$ women who had intramural myoma at single site independent to other subtypes developed menorrhagia,while menorrhagia was also present when intramural myoma coexisted with subserous( 11 out of 13), submucous (4 out of 5) and broad ligament (1 out of 1)myoma. On the other hand, broad ligament and subserous myoma that occurred independently did not produce menorrhagia (Table 2). Menorrhagia was present in almost all women with submucus myoma: two out of two women with solitary submucus myoma and 5 out of 6 women with submucus myoma which occurred in combination with intramural and subserous myoma. However, when tested for relationship with menorrhagia, myoma occurring at single site like submucous, intramural and subserous myoma did not significantly correlated with menorrhagia ( $p$-value $>0.05$ ). Similarly we could not find significant correlation between different types of myoma occurring in combinations with menorrhagia ( $p$-value $>0.05$ ). Although almost all submucus myoma except one produced menorrhagia, when tested for relationship with menorrhagia, all types of myoma, either at single site or occurring in combinations were not found to have any statistical significant correlation with menorrhagia (Chi-square test: $\mathrm{p}$-value $>0.05$ ).

The relative risk of occurrence of menorrhagia was 1.7 times in submucosal myoma in comparison to intramural myoma (Table 3). As subserous myoma did not produce menorrhagia, relative risk of menorrhagia in this type of myoma could not be evaluated.

Average "total area of all myoma in each case" was $63.2 \pm 80.0 \mathrm{~cm}^{2}$. Area of myoma was measured by multiplying length and breadth in $\mathrm{cm}^{2}$, which was summated in cases of multiple myoma. In our study, majority of myoma $36(51.4 \%)$ had cross-sectional area between $1-50 \mathrm{~cm}^{2}$. There was no statistical significant correlation between size of myoma and menorrhagia ( $p$-value $=0.19)$. Out of 70 cases $36(51.4 \%)$ cases had total area of myoma less than $50 \mathrm{~cm}^{2}$ and $26(37.1 \%)$ had area between $51 \mathrm{~cm}^{2}$ to $100 \mathrm{~cm}^{2}$. But occurrence of menorrhagia in these two groups was opposite (Table 4). Similarly, myoma of similar sizes behaved differently in relation to menorrhagia.

Out of 16 intramural myoma with area of $0.25-25 \mathrm{~cm}^{2}, 11$ $(68.75 \%)$ cases had menorrhagia, whereas out of 10 with area $>75 \mathrm{~cm}^{2}$ only $3(30 \%)$ had menorrhagia. Similarly, it was seen that not only submucous myoma of bigger than $75 \mathrm{~cm}^{2}$ area, but even small sized submucus myoma of area $0.25-25 \mathrm{~cm}^{2}$ produced menorrhagia. Different types of myoma occurring either at single location or in combination were categorized as per their sizes and analyzed for relationship with menorrhagia. However it also failed to show any statistical significant relationship between size of myoma and menorrhagia taking into account even the types of myoma (Chi-square test).

\section{Discussion}

The most common type of myoma was intramural $(91.43 \%)$, either occurring at single site only $(62.9 \%)$ or in combination with other types of myoma $(18.47 \%)$. Sulaiman et $\mathrm{al}^{6}$ in 2002 also reported the same result. In their study, 37 (74\%) patients among 50 patients with leiomyoma who underwent uterine artery embolization had intramural type of myoma. Being most common, intramural myoma were also found to be associated with subserous myoma (20\%), with submucus myoma (8.6\%), and even with broad ligament myoma (1.4\%).

Menorrhagia is usually diagnosed in a clinical context according to patient own perception of her loss as there is considerable difficulty in accessing the volume of menstrual blood loss in women presenting with menorrhagia ${ }^{6}$. Because of the differences in commercially available tampons, sanitary pads and cloths used by women and their different soaking capacities, it is difficult to measure the menstrual blood loss. In consequence, the diagnosis has to be made solely from the patients' history. But in our study this drawback was overcome by detail menstrual history with special emphasis on any changes in previous cycle, duration of menstrual flow, occurrence of vaginal 
bleeding associated with clots or bleeding lasting longer than 7 days etc.

It was found that Menorrhagia (58.6\%) is the most common presenting symptom in our study followed by dysmenorrhoea (37.48\%) and pelvic heaviness and continuous per vaginal bleeding (18.57\% each). The results are quite comparable to that reported in most of the other studies ${ }^{4,6,10,11}$. In a study by Sulaiman et $\mathrm{al}^{6}$ in 2003 in Scotland, $84 \%$ of patients with uterine myoma presented with menorrhagia. Wegeinka G et al ${ }^{5}$ in 2003 reported gushing type of vaginal bleeding in $46 \%$ of patients with myoma. Menorrhagia was the most common complaint: $35.6 \%$ in a study by Shamshad Begum $^{10}$ and $37.35 \%$ in a study by Rashida Hafiz et $\mathrm{al}^{11}$. Although the figure is quite high in our study in comparison to others (Shamshad Begum, Rashida Hafiz, Wegeinka), but comparable to that done by Sulaiman et al, who objectively measured the menstrual blood loss using alkaline haematin method.

In our study, women suffered from menorrhagia in average for nearly three years (34.68 \pm 37.87 months), and the duration of continuous bleeding was about 2 months $(2.29 \pm 2.16)$. This finding differs from the study by Rashida Hafiz et al $^{11}$, in which 50.98 percent of patients presented within a period of 6 months to 2 years after developing menorrhagia. In our study, it was found that women tolerated menorrhagia for nearly 3 years. This may be due to less educational status and low socioeconomic condition of our patients. However, as continuous vaginal bleeding is considered as some serious illness, women with continuous per vaginal bleeding came for medical consultation much more earlier $(\mathrm{p}<0.05)$.

Some review articles have suggested that submucosal myoma are the greatest source of leiomyoma related symptom $^{7}$ or heavy bleeding 9 . In contrast, we found that leiomyoma at some particular location were not significantly associated with menorrhagia ( $\mathrm{p}$ value $>0$. 05). Although almost all submucosal myoma presented with menorrhagia (7 out of 8), there was no significant correlation between these two variables.

Leiomyoma is thought to cause increased bleeding, exactly how do they do so is not clear. A common clinical hypothesis is that leiomyoma increase the surface of endometrium, then increasing the volume of material expelled during menstruation. An increased surface area could be caused by a submucosal myoma or a large myoma. Our results somehow support the above fact only in term of increased risk of menorrhagia in submucosal myoma, however there was no statistically significant correlation between type of myoma and menorrhagia ( $p$ value $>0.05$ ). The relative risk of menorrhagia was 1.7 times in submucosal myoma (RR:
1.74, 95\% Confidence interval: $1.42-2.14)$ in comparison to intramural myoma (RR: $0.77,95 \%$ Confidence interval: $0.53-1.13$ ), which means that submucosal myoma were associated with the increased risk of menorrhagia. The relative risk could not be evaluated in subserous myoma as they did not produce menorrhagia. Our findings are consistent with the previous studies ${ }^{4,6}$, but inconsistent with others ${ }^{5}$. Wegienka et al in 2003 evaluated in a bigger sample size (941 patients), including even the asymptomatic women. They used TAS and TVS for the confirmation of myoma. They stated that nonsubmucosal myoma was found to be associated with heavy bleeding to the same extent as submucosal ones. Marino et al in 2004 conducted a study in Washington taking also large sample size (341 Patients), using TVS for detection of leiomyoma reported that intramural and subserous myoma are unrelated to menstrual cycle characteristics. However in their study, due to less number of submucosal myoma no statistical analysis was performed for this type of myoma.

The major drawback of these two studies were of being subjective in nature, relying in terms of patients own perception of blood loss. None of them actually objectively measured the blood loss during menstruation. Our findings did not differ from the study by Sulaiman et $\mathrm{al}^{6}$. Although they also had certain limitations, like less sample size ( 50 patients only) and asymptomatic women were not included in the study, they used the best method of MRI for detection of myoma, their location and size along with the gold standard method of assessment of menstrual blood loss, i.e. the alkaline hematin technique. He found that MBL correlated neither with myoma size nor location, although all women with submucosal myoma presented with menorrhagia. The results are quite comparable to our study where almost all women with submucosal myoma presented with menorrhagia and the risk of menorrhagia was more in submucosal ones as mentioned above.

Regarding the size of myoma, Wegienka et $\mathrm{al}^{5}$ found that small leiomyoma were associated with increased risk of heavy bleeding and the risk increased with the size of myoma. Contrary to our expectations, we could not find significant correlation between menorrhagia and size of myoma $(p=0.192)$. Fifty nine percent of small sized myoma with total area of less than $50 \mathrm{~cm} \mathrm{x}$ $\mathrm{cm}$ presented with menorrhagia, where as $51.7 \%$ of larger myoma of size $51-100 \mathrm{~cm}^{2}$ did not present with menorrhagia. The relative risk of menorrhagia did not increase as the size of myoma increased. The relative risk of menorrhagia with size of myoma greater than 50 $\mathrm{cm}^{2}$, irrespective of type of myoma was about $0.75(95 \%$ confidence interval $=0.50-1.13)$ in relation to myoma of equal to or lesser than $50 \mathrm{~cm}^{2}$ size. In a study by 
Wegienka $\mathrm{G}$ et al, the sample size was quite large (941 pre-menopausal women) and myoma was confirmed by TVS and TAS; but it had certain limitations. Here the diameter of largest myoma was taken, which can not give the true picture of total burden of myoma and so the true relation between myoma size and menorrhagia. However, our results are similar to other studies $^{4,6}$. Different methods were used for accessing the size of myoma in various studies. Sulaiman et al used the diameter of largest myoma and used MRI for detecting myoma where as Marino et al used total volume of myoma in $\mathrm{cm} \mathrm{x} \mathrm{cm} \mathrm{x} \mathrm{cm}$, which was summated in cases of multiple myoma, and he also used TVS for the confirmation of myoma.

Our study on the other hand, though with less sample size, has several strengths. In our study, presence of myoma was confirmed by cut section of hysterectomy specimen which was later confirmed by histopathological examinations. Moreover, total area of myoma was taken which was measured by scale in $\mathrm{cm}^{2}$ and the areas were summated in cases of multiple myoma, which actually reflects the true size of myoma, and thus, the true correlation between menorrhagia and size of myoma.

\section{Conclusion}

Intramural myoma was the most common type of uterine myoma in our study population. Menorrhagia was the commonest presenting symptom of uterine myoma with an average duration of $34.7 \pm 37.9$ months. There were no statistically significant correlation between site and size of myoma and menorrhagia ( $\mathrm{p}$ value $>0.05$ ) However, all submucosal myoma were associated with increased risk of menorrhagia $(\mathrm{RR}=1.74)$.

\section{References}

1. Broder MS, Kanouse DE, Mittman BS, Bernstein SJ. The appropriateness of recommendations for hysterectomy. Obstet Gynecol 2000;95:199-205.

2. Trojnar-PM, Reron A. Uterine leiomyomas- risk factors. Ginekol Pol 2003;74:1572-7.

3. Hallberg L, Hogdahl AM, Nilsson L, Rybo G. Menstrual blood loss- a population study. Acta Obstet Gynecol Scand 1966;45:320-51.

4. Marino JL, Eskenazi B, Warner M, Samuels S, Vercellini P, Gavoni N, et al. Uterine leiomyoma and menstrual cycle characteristics in a population-based cohort study. Hum Reprod 2004;19:2350-6.

5. Wegienka G, Baird DD, Hertz-Picciotto I, Harlow SD, Steege JF, Hill MC, et al. Self-reported heavy bleeding associated with uterine leiomyomata. Obstet Gynecol 2003;101:431-7.

6. Sulaiman S, Khaund A, McMillan N, Moss J, Lumsden MA. Uterine fibroids- do size and location determine menstrual blood loss? Eur J Obstet Gynecol Reprod Biol 2004;115:85-9.

7. Pelage JP, Le Dref O, Soyer P, Kardache M, Dahan H, Abitbol M, et al. Fibroid-related menorrhagia: treatment with superselective embolization of the uterine arteries and midterm follow-up. Radiology 2000;215:428-31.

8. Spies JB, Cooper JM, Worthington-Kirsch R, Lipman JC, Mills BB, Benenati JF. Outcome of uterine embolization and hysterectomy for leiomyomas: results of a multicenter study. Am J Obstet Gynecol 2004;191:22-31.

9. McLucas B, Adler L, Perrella R. Uterine fibroid embolization: nonsurgical treatment for symptomatic fibroids. J Am Coll Surg 2001;192:95105.

10. Begum S, Khan S. Audit of leiomyoma uterus at Khyber Teaching Hospital Peshawar. J Ayub Med Coll Abbottabad 2004;16:46-9.

11. Hafiz R, Ali M, Ahmad M. Fibroid as a causative factor in menorrhagia and its management. Pakistan J Med Res 2003;42:90-6. 